\title{
Role of the plasma cascade systems in ischemia/reperfusion injury of bone
}

Shengye Zhang ${ }^{1,2}$, Carlos Wotzkow ${ }^{2}$, Anjan K. Bongoni ${ }^{3}$, Jane Shaw-Boden ${ }^{2}$, Mark Siegrist ${ }^{2}$, Adriano Taddeo ${ }^{2,4}$, Fabian Blank ${ }^{2,5}$, Willy Hofstetter ${ }^{2}$, Robert Rieben ${ }^{2}$

${ }^{1}$ Graduate School for Cellular and Biomedical Sciences, University of Bern, Bern, Switzerland

${ }^{2}$ Department of Clinical Research, University of Bern, Bern, Switzerland

${ }^{3}$ Immunology Research Centre, St. Vincent's Hospital, Melbourne, Australia

${ }^{4}$ Division of Plastic and Hand Surgery, Inselspital, Bern, Switzerland

${ }^{5}$ Pulmonary Medicine, Bern University Hospital, Bern, Switzerland

\section{Corresponding author:}

Robert Rieben

Department of Clinical Research, University of Bern

Murtenstrasse 50

CH-3008 Bern, Switzerland

Phone: +41316329669

Fax: +41316327594

Email: robert.rieben@dkf.unibe.ch 


\section{Abstract}

Ischemia/reperfusion (I/R) injury has been extensively studied in organs such as heart, brain, liver, kidney, and lung. As a vascularized organ, bone is known to be susceptible to I/R injury too, but the respective mechanisms are not well understood to date. We therefore hypothesized that, similar to other organs, plasma cascade-induced inflammation also plays a role in bone $\mathrm{I} / \mathrm{R}$ injury. Reperfusion injury in rat tibia was induced by unilateral clamping of the femoral artery and additional use of a tourniquet, while keeping the femoral vein patent to prevent venous congestion. Rats were subjected to $4 \mathrm{~h}$ ischemia and $24 \mathrm{~h}$ reperfusion. Deposition of complement fragment $\mathrm{C} 3 \mathrm{~b} / \mathrm{c}$ and fibrin as well as expression of tissue factor (TF), tissue plasminogen activator (tPA), plasminogen activator inhibitor-1 (PAI-1), and Eselectin was detected by immunohistochemistry. In plasma, the levels of high mobility group box1 (HMGB1) were measured by ELISA. The total level of complement in serum was assessed by the $\mathrm{CH} 50$ test. Our results show that deposition of $\mathrm{C} 3 \mathrm{~b} / \mathrm{c}$ was significantly increased with respect to healthy controls in cortical bone as well as in marrow of reperfused limbs. C3b/c deposition was also increased in cortical bone, but not in bone marrow, of contralateral limbs. Deposition of fibrin, as well as expression of PAI-1, was significantly increased in bone after ischemia and reperfusion, whereas expression of tPA was reduced. These differences were most prominent in vessels of bone, both in marrow and cortical bone, and both in reperfused and contralateral limbs. However, PAI-1, was only increased in vessels of reperfused cortical bone and there were no significant changes in expression of Eselectin. With respect to solid bone tissue, a significant increase of $\mathrm{C} 3 \mathrm{~b} / \mathrm{c}$ and fibrin deposition was shown in osteocytes, and for fibrin also in the bone matrix, in both contralateral and reperfused cortical bone compared with normal healthy controls. A slight expression of TF was visible in osteocytes of the normal healthy control group, while TF was not present in the experimental groups. Moreover, $\mathrm{CH} 50$ values in serum decreased over time 
and HMGB1 was significantly increased in plasma of animals at the end of reperfusion. We conclude that ischemia and reperfusion of bone leads to activation of the complement and coagulation systems and a downregulation of the fibrinolytic cascade. In the acute phase, a vascular inflammation induced by activation of the plasma cascade systems also occurs in the bone. This is similar to I/R injury of other vascularized organs and tissues.

Keywords: Bone, ischemia/reperfusion injury, complement, coagulation, fibrinolysis 


\section{Introduction}

As a vascularized organ, bone is known to be susceptible to ischemia/reperfusion (I/R) injury [1-3]. This can be the case in traumatic or non-traumatic conditions, for example in limb replantation, transplantation, but also in surgery on the extremities in general. All cells including osteoblasts, osteocytes, osteoclasts, chondrocytes, and bone marrow cells can be affected and involved in sterile inflammation during reperfusion injury, leading to bone disorder $[4,5]$. We therefore wanted to explore the mechanisms involved in bone I/R injury, focusing on the activation of the plasma cascade systems and the involvement of the bone vasculature.

The role of the complement system in I/R injury was documented and discussed before [6-8]. With respect to limb and bone I/R injury in particular, Austen et al. demonstrated complement activation initiated by natural antibody in a murine hind limb I/R injury model [9]. It was also described that complement activation is triggered and plays an important role in patients with major trauma [10]. Anaphylatoxins C3a and C5a can recruit macrophages and neutrophils to the damaged cell surface, and they are also involved in activation of osteoblasts and osteoclasts on broken surfaces after trauma [11, 12].

In addition to complement activation, the coagulation cascade plays a role in I/R injury [1315]. Fibrin, the final product of coagulation activation, as well as thrombotic markers like thrombin-antithrombin complexes (TAT) and D-dimers, was shown to be increased in hind limb I/R injury models [16, 17]. Coagulation disorders were also manifested following trauma, which may lead to end-organ failure due to irreversible ischemia [18]. Factor VIIactivating protease was activated by damaged cells in multiple trauma patients [19]. Tissue factor was released in lower extremity trauma patients and led to increased thrombin generation that was maintained for at least 5 weeks [20]. A hypercoagulant state was also shown in ischemic legs in patients with arterial diseases [21]. The enhanced procoagulant 
activity was also indicated by higher levels of plasminogen activator inhibitor 1 (PAI-1) and lower levels of tissue plasminogen activator (tPA) in the lower extremities requiring microsurgical reconstructions and in forearm I/R injury in patients [22, 23], respectively. An increase of PAI-1, combined with a decrease of tPA expression shifts healthy, antiinflammatory and anti-coagulant endothelium into a pro-coagulant and anti-fibrinolytic phenotype.

Generally, the above studies were associated with I/R injury of extremities, but little or no attention was paid to the effect of I/R injury on the bone itself. In the present study, therefore, I/R injury of the bone was studied specifically, and the underlying mechanisms were investigated with focus on the plasma cascades. The duration of the ischemic time was set according to a critical limb I/R injury model in rabbits reported by Hsieh et al [4]. These authors claimed that bone I/R injury was initiated within $2-4 \mathrm{~h}$ of ischemia by clamping the femoral artery and tourniquet application around the thigh of rabbit limbs. Therefore, we hypothesized that rat tibia bone responds to $4 \mathrm{~h}$ ischemia induced by occlusion of the femoral artery and additional use of a tourniquet, followed by $24 \mathrm{~h}$ reperfusion. Furthermore, we hypothesized that, similar to I/R injury of other vascularized organs or tissues, activation of the plasma cascade systems, including complement, coagulation, and fibrinolysis, is involved in I/R injury of the bone [24-26]. Our hypothesis may be limited in scope due to differences in techniques between this study and previous studies [4, 27, 28]. However, we were not attempting to create a "pure" model of bone $\mathrm{I} / \mathrm{R}$ injury but examine whether I/R injury to a rat hind limb similar to crush injury (Hsieh et al. [4]) may also, either directly or indirectly, affect bone specific mechanisms. 


\section{Materials and methods}

\subsection{Animals}

Male Wistar rats (wild type, bred at the central animal facility, University of Bern), weighing between $280 \mathrm{~g}$ and $320 \mathrm{~g}$, were used in this study. All animals were housed under standard conditions with water and food ad lib. All animal experiments were performed in accordance with the U.K. Animals Act (scientific procedures) and the NIH Guide for the Care and Use of Laboratory Animals, as well as the Swiss animal protection law. All animal studies complied with the ARRIVE guidelines. The animal experimentation committee of the cantonal veterinary service (Canton of Bern, Switzerland) approved all animal procedures, permission no. BE70/14. Experimental protocols were refined according to the $3 \mathrm{R}$ principles and stateof-the-art anesthesia and pain management were used to minimize the number of animals and reduce the exposure of the animals to stress and pain during the experiments.

\subsection{Surgical procedure}

Anesthesia was induced with $3 \%$ isoflurane in $50 \%$ oxygen / air and then maintained at $2 \%$ isoflurane using a standard rat nose mask (Provet, Lyssach, Switzerland). Analgesia was administered with $0.05 \mathrm{mg} / \mathrm{kg}$ of buprenorphine (Temgesic, Reckitt Benckiser, Switzerland AG), subcutaneously injected 30 min before induction of the surgical procedure. Our rat model was established according to our previous work [29-32] and rat vascular anatomy [33]. Rats were placed on a heating pad and the rectal temperature kept at $37 \pm 1^{\circ} \mathrm{C}$. Oxygen saturation, heart and breath rate, breath distention (for depths of anesthesia) and body temperature were monitored throughout the anesthesia using a Mouse-Ox plus System (Starr Life Sciences, Oakmont, PA, USA). The used rat model of crush I/R injury was established based on our previous work [29-32] and a study published by Hsieh et al. [4]. Following incision in the left groin, femoral artery, vein, and nerve were isolated from the surrounding 
tissue. The femoral artery was then clamped with two microvascular clamps (B1-V, S\&T, Neuhausen, Switzerland) proximal to the deep femoral artery branch. Meanwhile, a tourniquet, connected to a weight of $450 \mathrm{~g}$, was placed underneath the femoral vessels around the thigh as high as possible to block collateral blood supply, while keeping the femoral vein open. The animals were subjected to $4 \mathrm{~h}$ ischemia and $24 \mathrm{~h}$ reperfusion (by releasing the clamps and the tourniquet). The operation and the use of tourniquet are shown in Fig. 1. Upon reperfusion, the rats were allowed to wake up and another dose of buprenorphine was given to provide adequate analgesia. At the end of the $24 \mathrm{~h}$ reperfusion period, the rats were anesthetized again and sacrificed by exsanguination during organ removal in deep anesthesia. Blood and tibia bone samples were taken for further analysis.

Two groups were included in this study, the I/R injury group $(n=6)$ and normal, healthy rats as a controls $(n=5)$. The number of animals per group was calculated based on the NIH 'guidelines for the design and statistical analysis of experiments using laboratory animals' and the ARRIVE guidelines [34, 35]. Expected differences between groups were based on data on edema formation (wet/dry ratio) of a previous, as yet unpublished study using the same crush I/R injury model. In this study, wet/dry ratios differed by $1.2 \pm 0.4$ between the control animals and the group undergoing I/R injury. Sample size calculations based on these data resulted in five animals per group. 


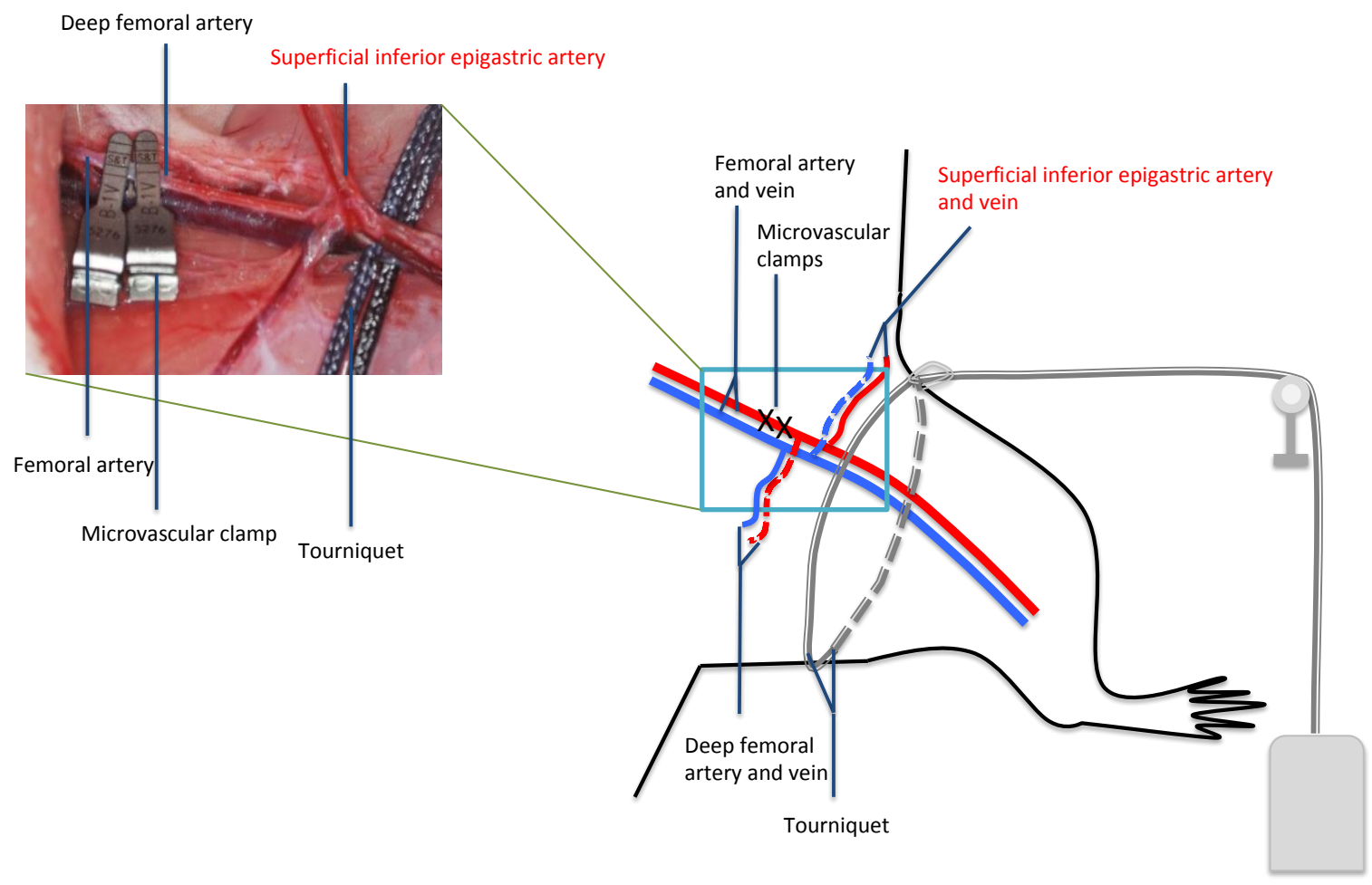

Fig. 1: Experimental induction of hind limb crush I/R injury in the rat. A photo of the microsurgery operation is shown on the left panel and a diagram of the rat vascular and microsurgery procedure is drawn on the right panel. In brief, after incision in the left groin, femoral artery, vein, and nerve were isolated. The femoral artery was then clamped with two microvascular clamps proximal to the deep femoral artery branch as shown on the photo and indicated by ' $\mathrm{XX}$ ' on the drawing. A tourniquet, connected to a weight of $450 \mathrm{~g}$, was placed underneath the femoral vessels around the thigh as high as possible to block collateral blood supply, while keeping the femoral vein open. Drawing is not to scale.

\subsection{Sample preparation}

Tibia bones from normal control rats and reperfused as well as contralateral hind limbs from the experimental group were dissected free from the surrounding soft tissue, rinsed with phosphate-buffered saline (PBS), blotted dry, and immediately fixed in 4\% paraformaldehyde for $24 \mathrm{~h}$ at room temperature. After that they were washed in tap water overnight and transferred into $70 \%$ ethanol until further use. When all bones were available, decalcification was performed in $15 \%$ EDTA / $0.5 \%$ paraformaldehyde and checked by X-ray. After 
complete decalcification, bone tissue was rinsed under tap water overnight and then dehydrated. Samples including transverse sections from the middle of the shaft were then embedded in paraffin and $4 \mu \mathrm{m}$ sections were cut. Blood was drawn at baseline, end of ischemia and end of reperfusion to collect EDTA- and citrate-plasma as well as serum. Plasma and serum samples were aliquoted and stored at $-80^{\circ} \mathrm{C}$ until analysis.

\subsection{Immunohistochemistry}

To assess the involvement of the complement, coagulation, and fibrinolytic cascades after $24 \mathrm{~h}$ reperfusion, immunohistochemistry (IHC) staining was performed. Briefly, sections were deparaffinized in xylene, rehydrated through graded alcohols, and washed in distilled $\mathrm{H}_{2} \mathrm{O}$. Then antigen retrieval was performed in $15 \mu \mathrm{g} / \mathrm{ml}$ of Proteinase K (Ref. 03115879001 , Roche Diagnostics, Indianapolis, IN, USA) in a water bath at $37^{\circ} \mathrm{C}$ for $5 \mathrm{~min}$. Endogenous peroxidase activity was blocked with $3 \% \mathrm{H}_{2} \mathrm{O}_{2}$ for 10 min. Primary antibodies were then applied to the sections in a humid chamber at $4{ }^{\circ} \mathrm{C}$ overnight. The following primary antibodies were used: rabbit anti-human C3b/c (A0062, Dako, Glostrup, Denmark), goat antirat tissue factor (TF) (sc-23596, Santa Cruz Biotechnology, Inc., Texas, USA), rabbit antihuman fibrinogen (A0080, Dako), rabbit anti-rat tPA (ab14198, Abcam, Cambridge, UK), goat anti-rat PAI-1 (SC-6644, Santa Cruz), and rabbit anti-human E-selectin (bs-1273, Bioss Inc., Woburn, Massachusetts, USA). Then affinity-purified, biotin-conjugated rabbit antigoat (E0466, Dako) and goat anti-rabbit (E0432, Dako) secondary antibodies were applied to all sections for 90 minutes at room temperature. Afterwards, sections were incubated with avidin-biotin-peroxidase complex (PK6100, Vectastain, Burlingame CA, USA) for 45 min. Bound peroxidase was detected by 3,3'-diaminobenzidine (DAB) substrate for 1-3 min in the dark. Finally, sections were counterstained with hematoxylin (GHS232, Sigma-Aldrich, St. Louis, MO, USA) and mounted in Aquatex (108562, Darmstadt, Germany). For visualization 
and acquisition, images were taken with a bright field microscope (Leica DMI 4000B, Leica Microsystems Schweiz, Heerbrugg, Switzerland) equipped with an HCX PL APO 63x 1.400.6 lens and a color camera (DFC425). For each marker, 3-5 representative images were taken per slide and used for quantification or scoring of staining intensity.

The intensity of chromogen stain in IHC was quantified within the vessels by Fiji software (ImageJ) (http://fiji.sc/Fiji). Vessels were identified visually and delineated by hand. The intensity of the staining was then calculated by Fiji software using the color deconvolution feature for $\mathrm{H}$ DAB staining. The stronger the staining visually, the weaker the intensity. This leads to an inverse correlation between the staining signal and the measured intensity. We followed the protocol published by Nguyen et al. using reciprocal intensity (subtract the measured intensity from the maximum intensity value of 255), which is proportional to the actual staining intensity [36]. Staining intensity of osteocytes and bone matrix was scored from $0-3(0=$ negative, $1=$ weak, $2=$ medium, $3=$ strong $)$ by eye based on the whole tissue section. The same sections were assigned grades from 1-5 (grade 1: 0-20\% of surface stained, - grade 5: $80-100 \%$ of surface or number of cells stained). Each section was then given an arbitrary value calculated as intensity $\times$ grading and these values were used for statistical evaluation.

\subsection{Hemolytic complement (CH50) assay}

Activity of the classical pathway in serum was analyzed using a standard hemolytic complement (CH50) assay. Packed sheep erythrocytes were washed in veronal buffered saline $\left(\mathrm{VBS}^{++}\right)$at 1:30 dilution until the supernatant was clear. The erythrocytes were then diluted to achieve $10^{9}$ cells $/ \mathrm{ml}$ and incubated with rabbit anti-sheep erythrocyte antibody (S1389, Sigma-Aldrich) for $20 \mathrm{~min}$ at $37^{\circ} \mathrm{C}$. After centrifugation, the antibody-sensitized 
erythrocytes were resuspended in Alsever's solution (A3551, Sigma-Aldrich) and kept at $4{ }^{\circ} \mathrm{C}$ overnight. On the second day, the cells were washed again and adjusted to $10^{8} / \mathrm{ml}$. Serum samples were then added to a transparent 96 well microplate (Nunc, Roskilde, Denmark) and incubated with the above adjusted cells at $37^{\circ} \mathrm{C}$ for $60 \mathrm{~min}$, then stopped with PBS. For the controls, erythrocytes were incubated in a veronal buffer $\left(\mathrm{DGVB}^{++}\right)$and the reaction stopped with water (T100, 100\% lysis) or PBS (T0, 0\% lysis, background). At the end, optical density of free hemoglobin was measured at 412nm (Infinite M1000 spectrophotometer, Tecan, Männedorf, Switzerland). A lysis percentage was calculated as: $\frac{O D \text { sample-OD T0 }}{O D T 100-O D T 0} * 100$.

\subsection{Measurement of plasma HMGB1 by ELISA}

High-mobility group box 1 protein (HMGB1) is a nuclear protein present in almost all eukaryotic cells. It can be secreted by activated macrophages, mature dendritic cells and natural killer cells and released into circulation as a proinflammatory mediator $[37,38]$. To determine HMGB1 in plasma, a homemade sandwich-ELISA was performed. In brief, a capture antibody, rabbit anti-rat HMGB1 (H9539; Sigma) and HRP-conjugated detection antibody, HRP conjugated rabbit anti-rat HMGB1 (ab128129; Abcam) were added, followed by SureBlue TMB Microwell Peroxidase Substrate (52-00-01, KPL, Gaithersburg, MD, USA) for color development and stopped by $1 \mathrm{~mol} / \mathrm{L} \mathrm{H}_{2} \mathrm{SO}_{4}$. At the end, optical density was measured at 450/540 $\mathrm{nm}$ using a FLUOstar Omega microplate reader (BMG LABTECH GmbH, Ortenberg, Germany).

\subsection{Statistical analysis}

All data are presented as mean \pm standard deviation (SD). Statistical analyses were performed by one-way analysis of variance (ANOVA) followed by Fisher's LSD post hoc test with 
GraphPad Prism 6 software (GraphPad, San Diego, CA, USA). P values $<0.05$ were considered statistically significant.

\section{Results}

\subsection{Deposition of $\mathrm{C} 3 \mathrm{~b} / \mathrm{c}$}

To assess activation of the complement cascade in reperfusion injury of bone, deposition of the central component fragment $\mathrm{C} 3 \mathrm{~b} / \mathrm{c}$ was analyzed. In vessels of cortical bone, $\mathrm{C} 3 \mathrm{~b} / \mathrm{c}$ deposition was significantly increased in both reperfused and contralateral limbs compared with normal, healthy controls $(\mathrm{p}=0.0055$ and $\mathrm{p}=0.0254$, respectively). In vessels of bone marrow, a significant increase of $\mathrm{C} 3 \mathrm{~b} / \mathrm{c}$ was shown in the reperfused, but not in the contralateral marrow $(\mathrm{p}=0.0037$ reperfused vs. contralateral and $\mathrm{p}=0.0077$ reperfused vs. normal, Fig. 2).

A

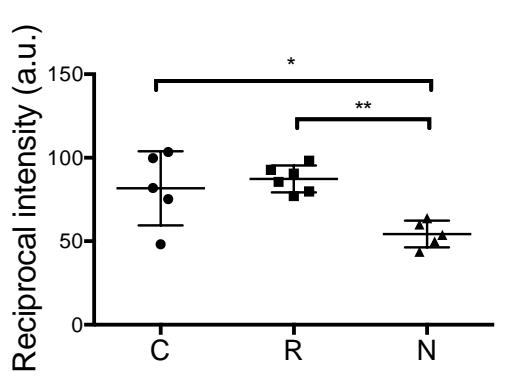

C

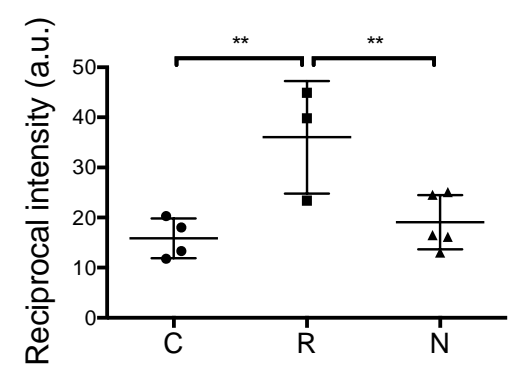

B

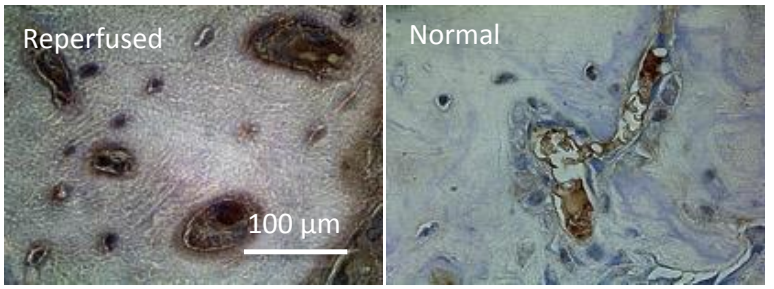

D

Marrow

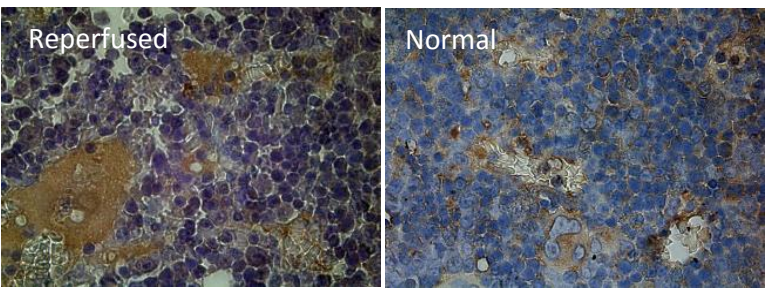

Fig. 2. Deposition of $\mathrm{C} 3 \mathrm{~b} / \mathrm{c}$ in bone tissue. A, quantitative comparison of $\mathrm{C} 3 \mathrm{~b} / \mathrm{c}$ deposition in vessels of cortical bone. $\mathrm{B}$, representative images for $\mathrm{C} 3 \mathrm{~b} / \mathrm{c}$ staining in reperfused and normal cortical bones from healthy, unmanipulated animals. $\mathrm{C}$, quantitative comparison of $\mathrm{C} 3 \mathrm{~b} / \mathrm{c}$ deposition in vessels of marrow. $\mathrm{D}$, representative 
images for $\mathrm{C} 3 \mathrm{~b} / \mathrm{c}$ staining in reperfused and normal marrow from healthy, unmanipulated animals. Data of individual experiments are represented by dots with indication of mean \pm SD by lines. Scale bar represents 100 $\mu \mathrm{m} . \mathrm{C}$ - contralateral; $\mathrm{R}$ - reperfused; $\mathrm{N}$ - normal. $* \mathrm{P}<0.05 ; * * \mathrm{P}<0.01$.

\subsection{Expression of tissue factor and deposition of fibrin}

To study the role of the coagulation cascade in bone I/R injury, IHC staining was performed for expression of $\mathrm{TF}$ and deposition of fibrin. $\mathrm{TF}$ expression was significantly raised in vessels of the contralateral bone marrow compared with normal controls without any forms of intervention $(\mathrm{p}=0.0422)$, but the increase in staining intensity was not statistically significant in reperfused bones. In the cortical bone, no significant differences were detected for expression of TF between groups (Fig. 3).

A

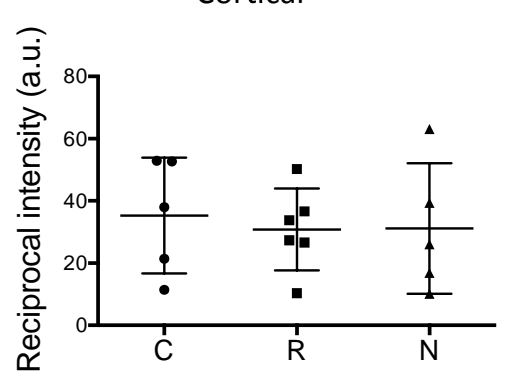

C

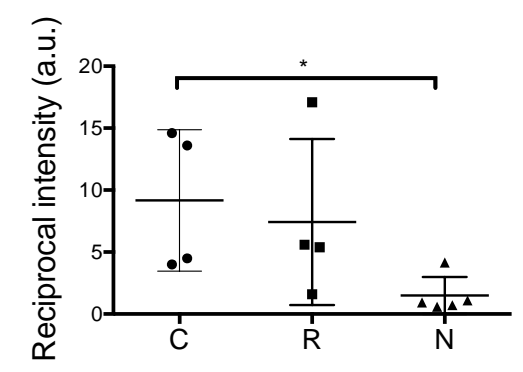

B

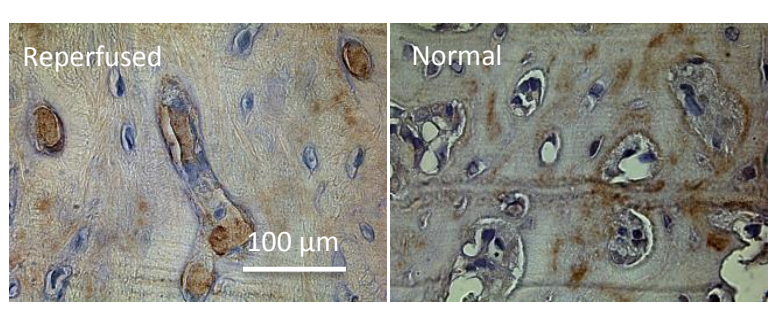

D

Marrow

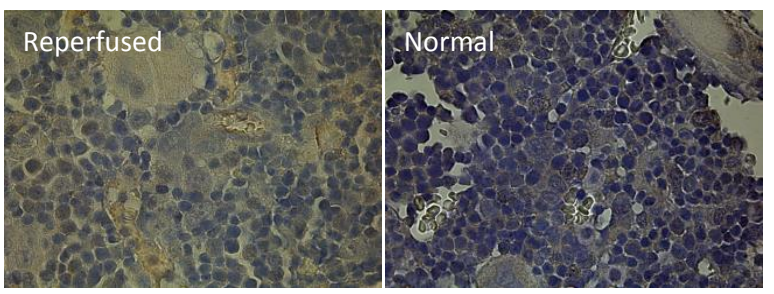

Fig. 3. Expression of tissue factor in bone tissue. A, quantitative comparison of TF expression in vessels of cortical bone. B, representative images for TF staining in reperfused and normal cortical bones from healthy, unmanipulated animals. C, quantitative comparison of TF expression in vessels of bone marrow. D, representative images for TF staining in reperfused and normal marrow from healthy, unmanipulated animals. 
Data of individual experiments are represented by dots with indication of mean \pm SD by lines. Scale bar represents $100 \mu \mathrm{m}$. $\mathrm{C}$ - contralateral; $\mathrm{R}$ - reperfused; $\mathrm{N}$ - normal. $* \mathrm{P}<0.05$.

Deposition of fibrin was significantly increased in vessels of both reperfused and contralateral cortical bones compared to normal ones without manipulation (both $\mathrm{p}<0.0001$ ), as well as in the respective bone marrow sections ( $\mathrm{p}=0.0024$ and $\mathrm{p}=0.0011$, respectively, Fig. 4).

A

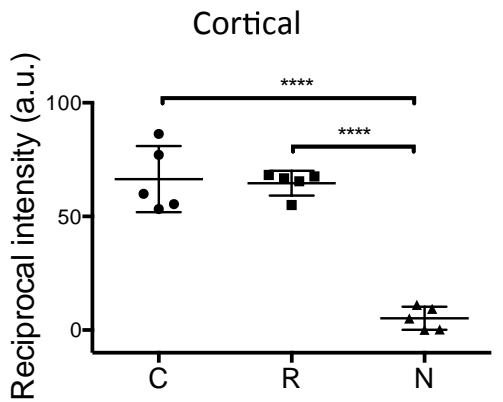

C

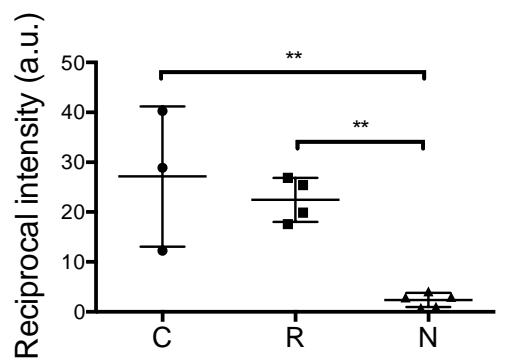

B

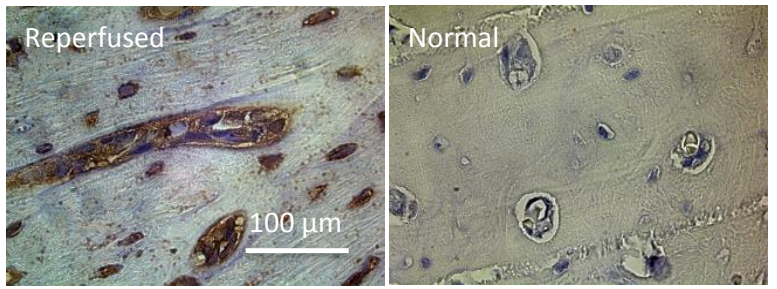

D

Marrow
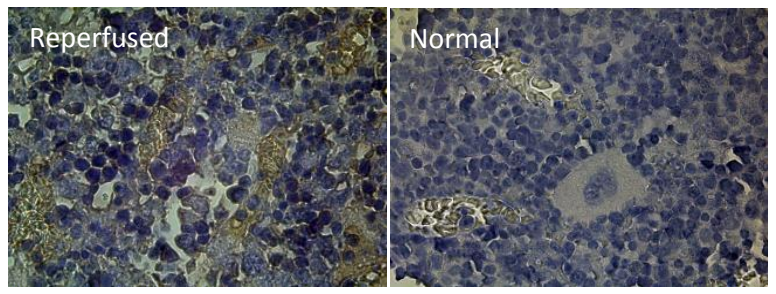

Fig. 4. Deposition of fibrin in bone tissue. A, quantitative comparison of fibrin deposition in vessels of cortical bone. B, representative images for fibrin staining in reperfused and normal cortical bones from healthy, unmanipulated animals. C, quantitative comparison of fibrin deposition in vessels of marrow. D, representative images for fibrin staining in reperfused and normal marrow from healthy, unmanipulated animals. Data of individual experiments are shown by dots with indication of mean \pm SD by lines. Scale bar represents $100 \mu \mathrm{m}$. $\mathrm{C}$ - contralateral; $\mathrm{R}$ - reperfused; $\mathrm{N}$ - normal. $* * \mathrm{P}<0.01 ; * * * * \mathrm{p}<0.0001$. 


\subsection{Expression of tPA and PAI-1}

To assess the involvement of the fibrinolytic system in bone I/R injury, expression of tPA and PAI-1 was evaluated by IHC. Expression of tPA was significantly down regulated in vessels of both reperfused and contralateral cortical bones as compared to healthy controls without intervention ( $\mathrm{p}=0.0063$ and $\mathrm{p}=0.0023$, respectively). Also in vessels of bone marrow, a significant downregulation of tPA expression was found both in the reperfused and contralateral limbs (both $\mathrm{p}<0.0001$, respectively, Fig. 5).

A

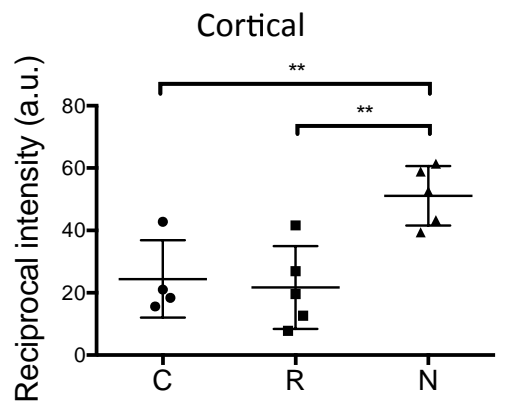

C

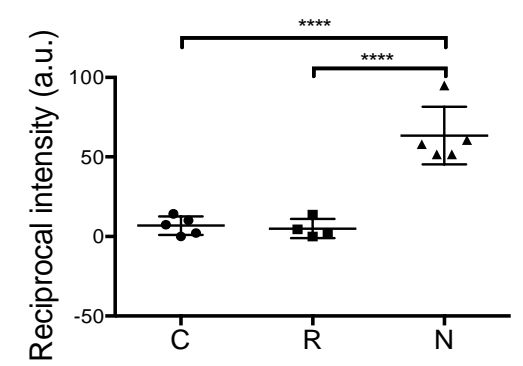

B

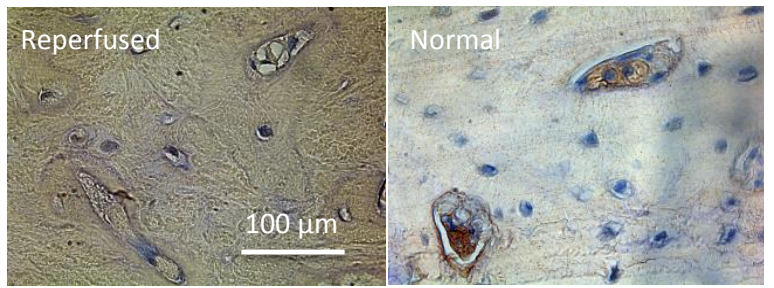

D

Marrow

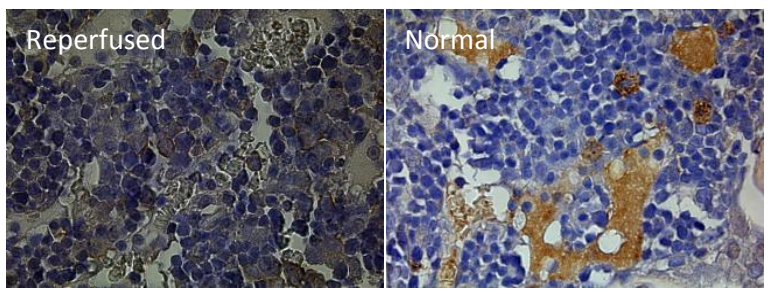

Fig. 5. Expression of tPA in bone tissue. A, quantitative comparison of tPA expression in vessels of cortical bone. B, representative images for tPA staining in reperfused and normal cortical bones from healthy, unmanipulated animals. C, quantitative comparison of tPA expression in vessels of marrow. D, representative images for tPA staining in reperfused and normal marrow from healthy, unmanipulated animals. Data of individual experiments are represented by dots with indication of mean \pm SD by lines. Scale bar represents 100 $\mu \mathrm{m} . \mathrm{C}$ - contralateral; R - reperfused; $\mathrm{N}$ - normal. $* * \mathrm{p}<0.01 ; * * * \mathrm{p}<0.0001$. 
PAI-1 expression was only visible in the vessels of cortical bone, not in bone marrow. Expression of PAI-1 was significantly up regulated in vessels of the reperfused cortical bones rather than the contralateral and normal, healthy controls $(\mathrm{p}=0.0117$ and $\mathrm{p}=0.0029$, respectively, Fig. 6).

A

Cortical

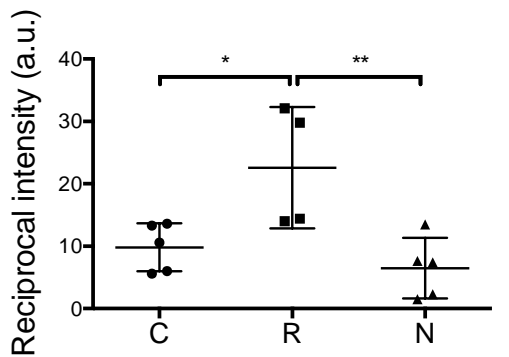

B

Cortical

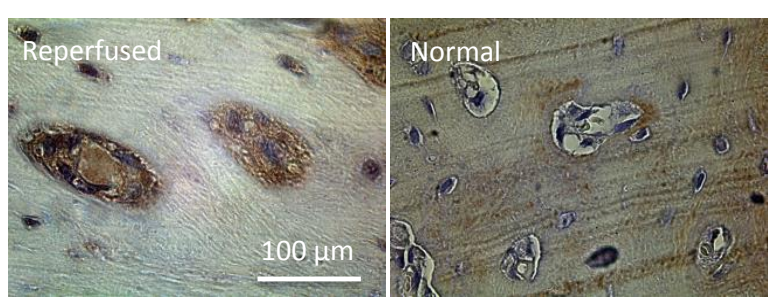

Fig. 6. Expression of PAI-1 in bone tissue. A, quantitative comparison of PAI-1 expression in vessels of cortical bone. B, representative images for PAI-1 staining in reperfused and normal cortical bones from healthy, unmanipulated animals. Data of individual experiments are represented by dots with indication of mean \pm SD by lines. Scale bar represents $100 \mu \mathrm{m}$. C - contralateral; R - reperfused; N - normal. *P<0.05; **p<0.01.

\subsection{Expression of E-selectin}

To evaluate expression of the adhesion molecule E-selectin on endothelial cells (ECs), IHC staining was performed. In a similar manner to PAI-1, E-selectin expression was only found in vessels of cortical bones, but not in bone marrow. No significant differences were observed for E-selectin expression between the reperfused cortical bones and unmanipulated, healthy bones ( $p=0.2354$, Fig. 7$)$. 
A

Cortical

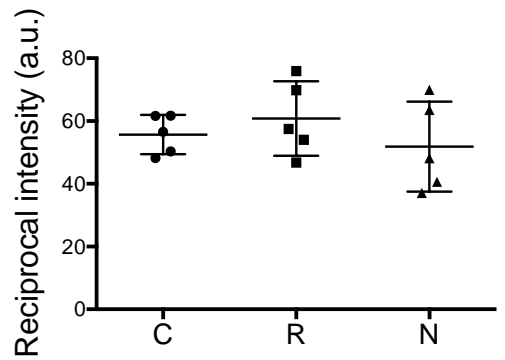

Cortical
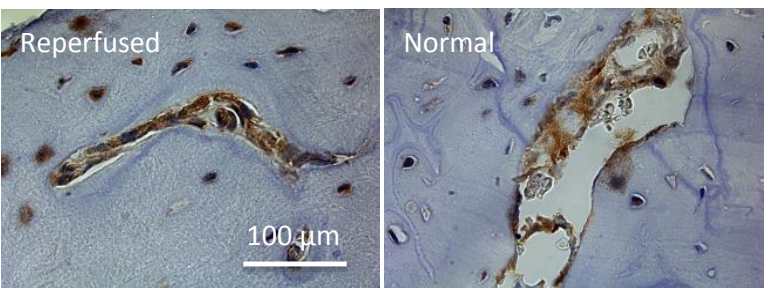

Fig. 7. Expression of E-selectin in bone tissue. A, quantitative comparison of E-selectin expression in vessels of cortical bone. B, representative images for E-selectin staining in reperfused and normal cortical bones from healthy, unmanipulated animals. Data of individual experiments are represented by dots with indication of mean \pm SD by lines. Scale bar represents $100 \mu \mathrm{m}$. C - contralateral; R - reperfused; $\mathrm{N}$ - normal.

\subsection{Overall effects of I/R injury on bone vessels, osteocytes and bone matrix}

To see the effects of I/R injury on bones including vasculature, osteocytes, and matrix, immunostaining of the above markers was evaluated. In Table 1 a summary of the effect of $\mathrm{I} / \mathrm{R}$ injury on the deposition or expression of plasma cascade proteins as well as E-selectin in bone vessels is shown.

\section{Table 1}

Effects of hind limb I/R injury on bone vasculature

\begin{tabular}{|c|c|c|c|c|}
\hline & \multicolumn{2}{|c|}{ Cortical bone } & \multicolumn{2}{|c|}{ Bone marrow } \\
\hline & Contralateral & Reperfused & Contralateral & Reperfused \\
\hline $\mathrm{C} 3 \mathrm{~b} / \mathrm{c}$ & $\uparrow$ & $\uparrow$ & $\uparrow$ & - \\
\hline $\mathrm{TF}$ & - & - & - & $\uparrow$ \\
\hline Fibrin & 个 & $\uparrow$ & $\uparrow$ & $\uparrow$ \\
\hline tPA & $\downarrow$ & $\downarrow$ & $\downarrow$ & $\downarrow$ \\
\hline PAI-1 & $\uparrow$ & - & Not detectable & Not detectable \\
\hline E-selectin & - & - & Not detectable & Not detectable \\
\hline
\end{tabular}


TF: tissue factor; tPA: tissue plasminogen activator; PAI-1: plasminogen activator inhibitor 1; $\uparrow$ : significantly increased compared with the normal healthy controls; $\downarrow$ : significantly decreased compared with the normal healthy controls; -: no significant differences between groups.

A specific analysis of immunohistochemistry staining of osteocytes and bone matrix was performed. Deposition of $\mathrm{C} 3 \mathrm{~b} / \mathrm{c}$ was significantly increased on osteocytes of the contralateral and reperfused bones, and fibrin was increased both on osteocytes and matrix of the contralateral and reperfused bones as compared with normal controls. A weak expression of TF was observed on osteocytes of the normal healthy rat tibias, while it was completely absent in the experimental groups. No significant differences were obtained for the other analyzed parameters. An overview of the presence of plasma cascade proteins on osteocytes and bone matrix is given in Table 2 and details are available in supplemental Fig. 1.

\section{Table 2}

Presence of plasma cascade proteins on osteocytes and matrix in cortical bone after hind limb I/R injury

\begin{tabular}{|c|c|c|c|c|c|c|}
\hline & & Osteocytes & & & Matrix & \\
\hline & Contralateral & Reperfused & Normal & Contralateral & Reperfused & Normal \\
\hline $\mathrm{C} 3 \mathrm{~b} / \mathrm{c}$ & $9.3 \pm 3.6^{*}$ & $11.0 \pm 4.1^{* *}$ & $3.6 \pm 0.5$ & $5.0 \pm 0.0$ & $5.2 \pm 0.4$ & $5.0 \pm 1.0$ \\
\hline $\mathrm{TF}$ & - & - & $1.4 \pm 0.9^{\# \# \#}$ & $2.3 \pm 1.5$ & $3.3 \pm 2.7$ & $5.6 \pm 3.0$ \\
\hline Fibrin & $12.0 \pm 2.5^{* * * * *}$ & $12.8 \pm 2.8^{* * * * *}$ & - & $7.2 \pm 1.3^{\text {***** }}$ & $7.0 \pm 2.5^{* * * * *}$ & - \\
\hline tPA & $2.5 \pm 1.4$ & $1.7 \pm 1.2$ & $1.2 \pm 1.3$ & $5.2 \pm 0.8$ & $6.5 \pm 1.2$ & $6.2 \pm 1.8$ \\
\hline PAI-1 & $1.0 \pm 1.5$ & $0.5 \pm 1.2$ & $0.6 \pm 1.3$ & $4.5 \pm 1.0$ & $5.5 \pm 0.8$ & $4.4 \pm 2.3$ \\
\hline
\end{tabular}

Data are arbitrary values (0-15) calculated as intensity $\times$ grading. For intensity, nothing=0; weak=1; mediate $=2$; strong=3. For field grading, $1-20 \%=1 ; 21-40 \%=2 ;>41-60 \%=3 ; 61-80 \%=4$ and $81-100 \%=5$. TF: tissue factor; tPA: tissue plasminogen activator; PAI-1: plasminogen activator inhibitor-1; -: absence of staining; *: $\mathrm{p}<0.05$ versus normal; **: $\mathrm{p}<0.01$ versus normal; ****: $\mathrm{p}<0.0001$ versus normal; \#\#\#: $\mathrm{p}<0.001$ versus contralateral and reperfused groups. 


\subsection{Serum complement activity}

In animals undergoing $4 \mathrm{~h}$ ischemia and $24 \mathrm{~h}$ reperfusion, serum complement activity was reduced over time. At baseline, the percentage of erythrocyte lysis was $96 \pm 3.6 \%$. After $2 \mathrm{~h}$ ischemia, values dropped to $89.3 \pm 2.8 \%$, which was significantly lower than baseline $(\mathrm{p}=0.0183)$. At the end of reperfusion, $85.9 \pm 6.0 \%$ erythrocyte lysis was measured ( $\mathrm{p}=0.0012$ vs. baseline, Fig. 8), suggesting complement consumption during the course of $\mathrm{I} / \mathrm{R}$ injury.

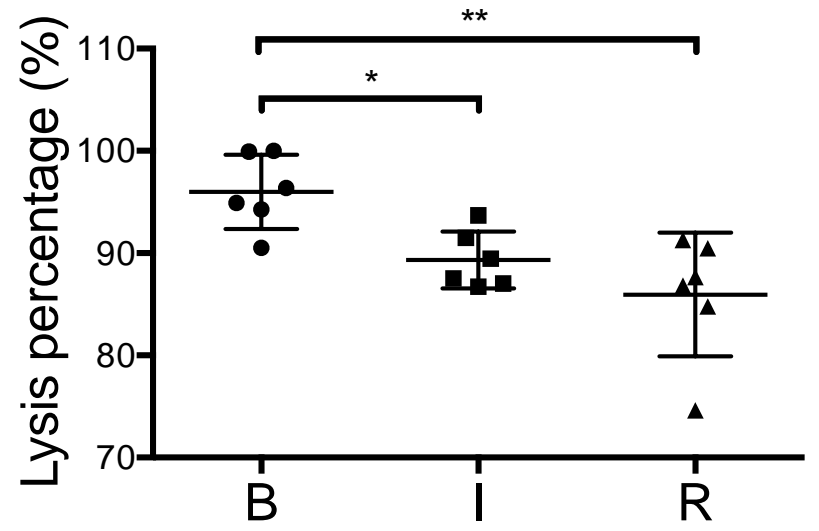

Fig. 8. Hemolytic complement activity by $\mathrm{CH} 50$ assay in serum at baseline, end of ischemia, and end of reperfusion. Data of individual experiments are represented by dots with indication of mean \pm SD by lines. B baseline; I - end of ischemia; $\mathrm{R}$ - end of reperfusion. $* \mathrm{P}<0.05 ; * * \mathrm{p}<0.01$.

\subsection{HMGB1 in plasma}

As an inflammatory marker, concentrations of HMGB1 in plasma were measured by ELISA. A significantly higher level of HMGB1 in plasma was found in animals subjected to hind limb I/R injury $(27.8 \pm 6.3 \mathrm{ng} / \mathrm{ml})$ compared with normal, healthy animals $(7.5 \pm 1.2 \mathrm{ng} / \mathrm{ml}$, p<0.0001, Fig. 9). 


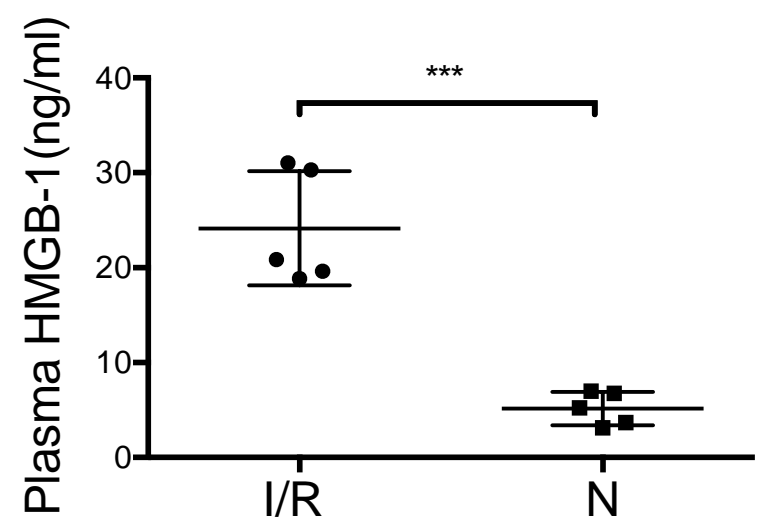

Fig. 9. Plasma levels of HMGB1 measured by ELISA. Data of individual experiments are represented by dots with indication of mean \pm SD by lines. I/R - ischemia/reperfusion injury group; $\mathrm{N}$ - normal control group. $* * * \mathrm{p}<0.0001$.

\section{Discussion}

The model of bone-related I/R crush injury induced in rat tibia was established on the basis of previous studies on I/R injury of rat hind limbs [31]. In a rat hind limb allotransplantation model routinely performed in our laboratory [32], the femoral arteries of both the donor and recipient rats are clamped at the same site as done in the present study. Bleeding of the femoral bone - which is cut during amputation - was never observed. Based on this experience and the anatomy of the rat vascular system [39], we can exclude the existence of functional collateral vessels nourishing the femoral bone of the contralateral limb upon unilateral clamping and tourniquet application. We therefore assume that our model leads to a complete, $4 \mathrm{~h}$ ischemia of the tibial bone. It was reported by others [4] using a bone chamber and intravital microscopy in rabbits, that an ischemia time of 2 to $4 \mathrm{~h}$ leads to a critical ischemia and reperfusion injury of cortical bone. From our own above mentioned studies [31] we also know that the limb is well reperfused as assessed by Laser Doppler, but a quantitative, specific analysis of bone reperfusion as performed in the rabbit bone chamber study [4] was not possible in our model. 
Our results show that activation of the plasma cascades, including complement, coagulation and fibrinolysis, may also play a role in I/R injury of bone. This is supported by increased deposition of $\mathrm{C} 3 \mathrm{~b} / \mathrm{c}$ and fibrin and increased expression of PAI-1, as well as reduced expression of tPA in bone vasculature from ischemic and reperfused rat hind limbs. A significant increase of $\mathrm{C} 3 \mathrm{~b} / \mathrm{c}$ and fibrin deposition was also shown on osteocytes (as well as matrix for fibrin) of both contralateral and reperfused hind limbs compared with normal, healthy controls.

The presence of fibrin deposits in the vasculature of contralateral bones suggests activation of the respective EC due to the systemic presence of pro-inflammatory mediators. Positive staining for fibrin of osteocytes and matrix in cortical bone was also observed in reperfused as well as contralateral limbs. This is most likely due to increased fibrinogen levels in plasma as described earlier for example in critical limb ischemia in patients [40]. One might speculate that osteocytes could also become activated due to $\mathrm{I} / \mathrm{R}$ injury and produce fibrinogen, but this remains to be analyzed in more detail. Deposition of fibrin in the contralateral limb was also found in an earlier study by our group, in which I/R injury of the gastrocnemius muscle was investigated in the same rat model [31]. In fact, remote organ damage caused by limb I/R injury has been shown by us [31] and others [27, 28, 41] in experimental models as well as clinical studies $[42,43]$.

Transition of bone vascular EC to a pro-coagulant and anti-fibrinolytic phenotype was further evidenced by downregulation of tPA, which activates plasmin to break down emerging fibrin clots. Downregulation of tPA expression, measured as impaired release of tPA into the circulation, was also reported in an extremity I/R injury study in humans [23]. PAI-1, a procoagulant marker, plays a role in I/R injury and it was reported previously that PAI-1 deficient mice show reduced fibrin deposition and a lower degree of inflammation in a lung I/R injury model [44]. Our data that tPA and PAI-1 expression are elevated in the bone tissue 
are consistent with results on blood levels of tPA and PAI-1 in patients requiring lower extremity microsurgical vascular reconstructions [22, 23].

EC activation is often associated with expression of adhesion molecules like E-selectin. In our study E-selectin expression was not significantly upregulated after $\mathrm{I} / \mathrm{R}$ injury. This finding is in line with a clinical study on elective limb surgery, in which no increase of soluble E-selectin was found in the blood of the patients [45]. However, an increased systemic release of endothelial adhesion molecules was reported in an amputation study with patients suffering from critical limb ischemia [46].

As an initiator of the coagulation pathway, TF is normally located in the sub-endothelium but can be induced on the surface of ECs due to vascular injury. There were no significant differences for vascular $\mathrm{TF}$ expression except a slight increase in the contralateral bone marrow. This could mean two things: Either fibrin formation may occur independently of TF expression, mainly via contact activation, or we miss a peak of TF expression that might occur earlier than $24 \mathrm{~h}$ after reperfusion.

In addition to coagulation, the complement system was also activated in our rat hind limb $\mathrm{I} / \mathrm{R}$ injury model. This was shown by a significant increase of $\mathrm{C} 3 \mathrm{~b} / \mathrm{c}$ deposits in vessels of cortical bone and bone marrow of reperfused limbs, as well as vessels of cortical bone of the contralateral limbs. C3b/c deposition was also increased on osteocytes of reperfused and contralateral bones as compared to normal controls. In line with these findings, we observed a significant reduction of functional complement in serum as reflected by $\mathrm{CH} 50$ values. This complement activation is consistent with clinical studies of critical limb ischemia [47, 48]. However, damage to skeletal muscle tissue is also occurring in our model (supplemental Fig. 1 and 2), which may also contribute to systemic complement activation and-consumption. 
In some models of I/R injury, complement activation was shown to be linked to recognition of ischemia-induced neoepitopes by natural antibodies $[49,50]$. However, in contrast to myocardial I/R injury [51], skeletal muscle reperfusion injury was not attenuated in the rat hind limb model by complement inhibition alone [31]. It remains to be determined whether in case of bone I/R injury EC activation relies on complement activation or not. In fact, EC can be activated by HMGB1, independent of complement activation, as shown recently by our group [52]. We found highly increased levels of HMGB1 in circulation after 24h of reperfusion, in line with a porcine poly-trauma model (including tibia fracture, lung contusion, and liver laceration) [53]. We might therefore speculate that EC activation in the bone vasculature is mediated - at least in part - by HMGB1. Yet, higher levels of HMGB1 are probably caused by extensive injury to the skeletal muscle tissue in our model (supplemental Fig. 2-3). This makes our experiments tentative due to the complexity of our animal model, i.e. ligating of the femoral artery and all the muscle tissue with associated veins and muscle tissue.

Another limitation of our study is the relatively short $-24 \mathrm{~h}-$ duration of reperfusion. I/R injury of bone may lead to healing and remodeling processes, which could not be studied in the current model. Due to technical difficulties with the bone sections we were also unable to reliably assess the staining of osteoblasts for the different markers of I/R injury and we had to limit the analysis of solid bone to osteocytes and bone matrix.

In conclusion, our results show that $\mathrm{I} / \mathrm{R}$ injury of bone induced by arterial clamping and additional tourniquet application leads to activation of the coagulation and complement cascades and a downregulation of the fibrinolytic system. In the acute phase, a vascular inflammation induced by activation of the plasma cascade systems seems to occur in bone. This is similar to events following I/R injury of other vascularized organs and tissues. 
However, our conclusions are limited by the fact that crush injury effects may also be present, which make interpretation more complicated.

\section{Funding}

Swiss National Science Foundation (SNSF), grants no. 32003B_135272 and 320030_156193 to RR and the SNSF Fellowship: P2BEP3_155459 to AKB. The China Scholarship Council (CSC) awarded grant support to SZ in Switzerland.

\section{Acknowledgements}

We thank Prof. Anne Angelillo Scherrer, University of Bern, for helpful discussion and critical comments. Many thanks also to Dr. Sidney G. Shaw, University of Bern, for proofreading and corrections. This study was support by the Microscopy Imaging Center (MIC), University of Bern. 


\section{References}

[1] A. Sckell, T. J. Demhartner, C. R. Fraitzl, M. Leunig. Reperfusion injury in free bone grafts after tourniquet-induced ischemia. Clin Orthop Relat Res 2003: 306-16.

[2] Y. Tamura, G. Inoue, T. Miura, N. Ishiguro, T. Shimizu. Reperfusion injury in bone: effects of CV-3611, a free radical scavenger, on ischemic revascularized bone grafts in rats. J Reconstr Microsurg 1992;8: 471-9.

[3] H. de Groot. Ischemia-reperfusion injury. A phenomenon, that also occurs in the bone. Mat.-wiss. u. Werkstofftech 2007;38.

[4] A. S. Hsieh, H. Winet, J. Y. Bao, H. Glas, H. Plenk. Evidence for reperfusion injury in cortical bone as a function of crush injury ischemia duration: a rabbit bone chamber study. Bone 2001;28: 94-103.

[5] M. Sato, N. Sugano, K. Ohzono, S. Nomura, Y. Kitamura, Y. Tsukamoto, S. Ogawa. Apoptosis and expression of stress protein (ORP150, HO1) during development of ischaemic osteonecrosis in the rat. J Bone Joint Surg Br 2001;83: 751-9.

[6] T. V. Arumugam, I. A. Shiels, T. M. Woodruff, D. N. Granger, S. M. Taylor. The role of the complement system in ischemia-reperfusion injury. Shock 2004;21: 401-9.

[7] T. V. Arumugam, T. Magnus, T. M. Woodruff, L. M. Proctor, I. A. Shiels, S. M. Taylor. Complement mediators in ischemia-reperfusion injury. Clin Chim Acta 2006;374: 33-45.

[8] N. C. Riedemann, P. A. Ward. Complement in ischemia reperfusion injury. Am J Pathol 2003;162: 363-7.

[9] W. G. Austen, Jr., M. Zhang, R. Chan, D. Friend, H. B. Hechtman, M. C. Carroll, F. D. Moore, Jr. Murine hindlimb reperfusion injury can be initiated by a self-reactive monoclonal IgM. Surgery 2004;136: 401-6.

[10] M. T. Ganter, K. Brohi, M. J. Cohen, L. A. Shaffer, M. C. Walsh, G. L. Stahl, J. F. Pittet. Role of the alternative pathway in the early complement activation following major trauma. Shock 2007;28: 29-34.

[11] M. Huber-Lang, A. Kovtun, A. Ignatius. The role of complement in trauma and fracture healing. Semin Immunol 2013;25: 73-8.

[12] M. Huber-Lang, A. Ignatius, R. E. Brenner. Role of Complement on Broken Surfaces After Trauma. Adv Exp Med Biol 2015;865: 43-55.

[13] X. S. Zhou, Y. F. Qiao, R. P. Wu, R. S. Li. Study on related indexes of the coagulation and fibrinolytic system after renal ischemia reperfusion injury in Wistar rats. Saudi Med J 2013;34: 579-83.

[14] J. H. Erlich, E. M. Boyle, J. Labriola, J. C. Kovacich, R. A. Santucci, C. Fearns, E. N. Morgan, W. Yun, T. Luther, O. Kojikawa, T. R. Martin, T. H. Pohlman, E. D. Verrier, N. Mackman. Inhibition of the tissue factor-thrombin pathway limits infarct size after myocardial ischemia-reperfusion injury by reducing inflammation. Am J Pathol 2000;157: 1849-62.

[15] Y. Kobayashi, N. Yoshimura, K. Nakamura, H. Yamagishi, T. Oka. Expression of tissue factor in hepatic ischemic-reperfusion injury of the rat. Transplantation 1998;66: 708-16.

[16] C. Duehrkop, J. Denoyelle, S. Shaw, R. Rieben. Use of dextran sulfate in tourniquetinduced skeletal muscle reperfusion injury. J Surg Res 2014;187: 150-61.

[17] M. F. Conrad, D. H. Stone, H. Albadawi, H. T. Hua, F. Entabi, M. C. Stoner, M. T. Watkins. Local inflammatory and thrombotic responses differ in a murine model of partial and complete hindlimb ischemia/reperfusion. Surgery 2005;138: 375-81.

[18] D. J. Dries. Activation of the clotting system and complement after trauma. New Horiz 1996;4: 276-88.

[19] S. M. Kanse, A. Gallenmueller, S. Zeerleder, F. Stephan, O. Rannou, S. Denk, M. Etscheid, G. Lochnit, M. Krueger, M. Huber-Lang. Factor VII-activating protease is activated in multiple trauma patients and generates anaphylatoxin C5a. J Immunol 2012;188: 2858-65.

[20] J. M. Walenga, P. C. Kaiser, M. M. Prechel, D. Hoppensteadt, W. P. Jeske, F. Misselwitz, P. Bacher, M. R. Lassen, J. Fareed. Sustained release of tissue factor following thrombosis of lower limb trauma. Clin Appl Thromb Hemost 2014;20: 678-86. 
[21] S. A. Ray, M. R. Rowley, A. Loh, S. A. Talbot, D. H. Bevan, R. S. Taylor, J. A. Dormandy. Hypercoagulable states in patients with leg ischaemia. Br J Surg 1994;81: 811-4.

[22] E. Olsson, N. Svartling, S. Asko-Seljavaara, R. Lassila. Activation of coagulation and fibrinolysis in microsurgical reconstructions in the lower extremities. Br J Plast Surg 2001;54: 597-603.

[23] C. M. Pedersen, G. Barnes, M. R. Schmidt, H. E. Botker, R. K. Kharbanda, D. E. Newby, N. L. Cruden. Ischaemia-reperfusion injury impairs tissue plasminogen activator release in man. Eur Heart J 2012;33: 1920-7.

[24] M. N. Busche, G. L. Stahl. Role of the complement components C5 and C3a in a mouse model of myocardial ischemia and reperfusion injury. Ger Med Sci 2010;8.

[25] B. H. Heijnen, I. H. Straatsburg, N. D. Padilla, G. J. Van Mierlo, C. E. Hack, T. M. Van Gulik. Inhibition of classical complement activation attenuates liver ischaemia and reperfusion injury in a rat model. Clin Exp Immunol 2006;143: 15-23.

[26] B. de Vries, S. J. Walter, C. J. Peutz-Kootstra, T. G. Wolfs, L. W. van Heurn, W. A. Buurman. The mannose-binding lectin-pathway is involved in complement activation in the course of renal ischemia-reperfusion injury. Am J Pathol 2004;165: 1677-88.

[27] M. M. Yassin, D. W. Harkin, A. A. Barros D'Sa, M. I. Halliday, B. J. Rowlands. Lower limb ischemia-reperfusion injury triggers a systemic inflammatory response and multiple organ dysfunction. World J Surg 2002;26: 115-21.

[28] D. W. Harkin, A. Barros D'sa A, K. McCallion, M. Hoper, M. I. Halliday, F. C. Campbell. Circulating neutrophil priming and systemic inflammation in limb ischaemia-reperfusion injury. Int Angiol 2001;20: 78-89.

[29] J. M. Sacks, Y. R. Kuo, E. K. Horibe, T. Hautz, K. Mohan, I. L. Valerio, W. P. Lee. An optimized dual-surgeon simultaneous orthotopic hind-limb allotransplantation model in rats. J Reconstr Microsurg 2012;28: 69-75.

[30] R. Sucher, R. Oberhuber, C. Margreiter, G. Rumberg, R. Jindal, W. P. Lee, R. Margreiter, J. Pratschke, S. Schneeberger, G. Brandacher. Orthotopic hind-limb transplantation in rats. J Vis Exp 2010.

[31] C. Duehrkop, Y. Banz, R. Spirig, S. Miescher, M. W. Nolte, M. Spycher, R. A. Smith, S. H. Sacks, R. Rieben. C1 esterase inhibitor reduces lower extremity ischemia/reperfusion injury and associated lung damage. PLoS One 2013;8: e72059.

[32] T. Gajanayake, R. Olariu, F. M. Leclere, A. Dhayani, Z. Yang, A. K. Bongoni, Y. Banz, M. A. Constantinescu, J. M. Karp, P. K. Vemula, R. Rieben, E. Vogelin. A single localized dose of enzymeresponsive hydrogel improves long-term survival of a vascularized composite allograft. Sci Transl Med 2014;6: 249ra110.

[33] W.J. Revell M. Brookes. Blood supply of bone. 1998: pp. 10-11.

[34] M. F. Festing, D. G. Altman. Guidelines for the design and statistical analysis of experiments using laboratory animals. ILAR J 2002;43: 244-58.

[35] C. Kilkenny, W. J. Browne, I. C. Cuthill, M. Emerson, D. G. Altman. Improving bioscience research reporting: The ARRIVE guidelines for reporting animal research. J Pharmacol Pharmacother 2010;1: 94-9.

[36] D.H. Nguyen, T. Zhou, J. Shu, J. Mao. Quantifying chromogen intensity in immunohistochemistry via reciprocal intensity Protocol exchange -Nature 2013.

[37] C. Semino, G. Angelini, A. Poggi, A. Rubartelli. NK/iDC interaction results in IL-18 secretion by DCs at the synaptic cleft followed by NK cell activation and release of the DC maturation factor HMGB1. Blood 2005;106: 609-16.

[38] M. T. Lotze, K. J. Tracey. High-mobility group box 1 protein (HMGB1): nuclear weapon in the immune arsenal. Nat Rev Immunol 2005;5: 331-42.

[39] W.J. Revell M. Brookes. Blood supply of bone. British library cataloguing in publication data: Butterworth Scientific ltd; 1998.

[40] K. R. Woodburn, A. Rumley, G. D. Lowe, J. G. Pollock. Fibrinogen and markers of fibrinolysis and endothelial damage following resolution of critical limb ischaemia. Eur J Vasc Endovasc Surg 1995;10: 272-8. 
[41] M. D. Bianco-Batlles, A. Sosunov, R. A. Polin, V. S. Ten. Systemic inflammation following hind-limb ischemia-reperfusion affects brain in neonatal mice. Dev Neurosci 2008;30: 367-73.

[42] L. N. Lin, L. R. Wang, W. T. Wang, L. L. Jin, X. Y. Zhao, L. P. Zheng, L. D. Jin, L. M. Jiang, X. Q. Xiong. Ischemic preconditioning attenuates pulmonary dysfunction after unilateral thigh tourniquet-induced ischemia-reperfusion. Anesth Analg 2010;111: 539-43.

[43] W. K. Edrees, L. L. Lau, I. S. Young, M. G. Smye, K. R. Gardiner, B. Lee, R. J. Hannon, C. V. Soong. The effect of lower limb ischaemia-reperfusion on intestinal permeability and the systemic inflammatory response. Eur J Vasc Endovasc Surg 2003;25: 330-5.

[44] C. L. Lau, Y. Zhao, J. Kim, I. L. Kron, A. Sharma, Z. Yang, V. E. Laubach, J. Linden, G. Ailawadi, D. J. Pinsky. Enhanced fibrinolysis protects against lung ischemia-reperfusion injury. J Thorac Cardiovasc Surg 2009;137: 1241-8.

[45] A. Seekamp, M. Jochum, M. Ziegler, M. van Griensven, M. Martin, G. Regel. Cytokines and adhesion molecules in elective and accidental trauma-related ischemia/reperfusion. J Trauma 1998;44: 874-82.

[46] D. J. Newton, F. Khan, G. Kennedy, J. J. Belch. Improvement in systemic endothelial condition following amputation in patients with critical limb ischemia. Int Angiol 2008;27: 40812.

[47] M. Martin, A. Gottsater, P. M. Nilsson, T. E. Mollnes, B. Lindblad, A. M. Blom. Complement activation and plasma levels of C4b-binding protein in critical limb ischemia patients. J Vasc Surg 2009;50: 100-6.

[48] A. Bengtson, P. Holmberg, M. Heideman. The ischaemic leg as a source of complement activation. Br J Surg 1987;74: 697-700.

[49] M. Zhang, E. M. Alicot, I. Chiu, J. Li, N. Verna, T. Vorup-Jensen, B. Kessler, M. Shimaoka, R. Chan, D. Friend, U. Mahmood, R. Weissleder, F. D. Moore, M. C. Carroll. Identification of the target self-antigens in reperfusion injury. J Exp Med 2006;203: 141-52.

[50] L. Kulik, S. D. Fleming, C. Moratz, J. W. Reuter, A. Novikov, K. Chen, K. A. Andrews, A. Markaryan, R. J. Quigg, G. J. Silverman, G. C. Tsokos, V. M. Holers. Pathogenic natural antibodies recognizing annexin IV are required to develop intestinal ischemia-reperfusion injury. J Immunol 2009;182: 5363-73.

[51] Y. Banz, O. M. Hess, S. C. Robson, E. Csizmadia, D. Mettler, P. Meier, A. Haeberli, S. Shaw, R. A. Smith, R. Rieben. Attenuation of myocardial reperfusion injury in pigs by Mirococept, a membrane-targeted complement inhibitor derived from human CR1. Cardiovasc Res 2007;76: 482-93.

[52] A. K. Bongoni, N. Klymiuk, E. Wolf, D. Ayares, R. Rieben, P. J. Cowan. Transgenic Expression of Human Thrombomodulin Inhibits HMGB1-Induced Porcine Aortic Endothelial Cell Activation. Transplantation 2016.

[53] K. Horst, F. Hildebrand, R. Pfeifer, S. Hubenthal, K. Almahmoud, M. Sassen, T. Steinfeldt, H. Wulf, S. Ruchholtz, H. C. Pape, D. Eschbach. Impact of haemorrhagic shock intensity on the dynamic of alarmins release in porcine poly-trauma animal model. Eur J Trauma Emerg Surg 2016;42: 67-75. 


\begin{tabular}{|c|c|c|c|c|}
\hline & \multicolumn{2}{|c|}{ Cortical bone } & \multicolumn{2}{|c|}{ Bone marrow } \\
\hline & Reperfused & Contralateral & Reperfused & Contralateral \\
\hline Complement C3b/c & $\uparrow$ & $\uparrow$ & $\uparrow$ & - \\
\hline Tissue factor & - & - & - & $\uparrow$ \\
\hline Fibrin & $\uparrow$ & $\uparrow$ & $\uparrow$ & $\uparrow$ \\
\hline $\begin{array}{l}\text { Tissue plasminogen } \\
\text { activator (tPA) }\end{array}$ & $\downarrow$ & $\downarrow$ & $\downarrow$ & $\downarrow$ \\
\hline $\begin{array}{l}\text { Plasminogen } \\
\text { activator inhibitor } 1 \\
(\mathrm{PAI}-1)\end{array}$ & $\uparrow$ & 一 & $\begin{array}{c}\text { Not } \\
\text { detectable }\end{array}$ & $\begin{array}{c}\text { Not } \\
\text { detectable }\end{array}$ \\
\hline E-selectin & - & - & $\begin{array}{c}\text { Not } \\
\text { detectable }\end{array}$ & $\begin{array}{c}\text { Not } \\
\text { detectable }\end{array}$ \\
\hline
\end{tabular}

Graphical abstract 